\title{
miR-98 is involved in missed abortion by targeting GDF6 and FAPP2
}

\author{
Qi Zhu'1,2, Yi-Chao Dong1,2, Lu Zhang ${ }^{1,2}, \mathrm{Xu} \mathrm{Ma}^{1,2}$ and Hong-Fei Xia ${ }^{1,2}$ \\ ${ }^{1}$ Reproductive and Genetic Center of National Research Institute for Family Planning, Beijing, China and ${ }^{2}$ Graduate \\ Schools, Peking Union Medical College, Beijing, China
}

Correspondence should be addressed to X Ma or H-F Xia; Email: genetic@263.net.cn or hongfeixia@126.com

\begin{abstract}
Missed abortion (MA) is a common disease in obstetrics and gynecology. More and more studies have focused on the relationship between miRNAs and pregnancy maintenance and its related diseases. The aim of this article is to explore the relationship between miRNA and MA. The expression of miR-98 were detected by in situ hybridization and real-time PCR. Cell proliferation, activity and migration were measured via Edu, MTT, and transwell assays. The target genes of $\mathbf{m i R}-\mathbf{9 8}$ are identified by dual-luciferase activity assay. And the expression levels of target genes were determined by Western blot, real-time PCR and immunohistochemistry. miR-98 was significantly up-regulated in placental villi from over 35 years old MA patients compared with the age-matched normal pregnant women. Up-regulation of miR-98 suppressed the proliferation, activity and migration of the human trophoblast HTR-8/SVneo cell in vitro. miR-98 could bind to GDF6 and FAPP2 mRNA $3^{\prime}$-UTR and negatively regulate their expression. The downregulation of $m i R-98$ promoted cell proliferation, then knockdown of GDF6 or FAPP2 inhibited miR-98-mediated cell proliferation. GDF6 and FAPP2 expression in the placental villi from MA patients were decreased compared to normal placental tissues. The expression of $m i R-98$ in MA had an opposite relationship with the expression of GDF6 and FAPP2. Overexpression of miR-98 is associated with the occurrence of MA. miR-98 prevents proliferation, viability and migration of trophoblast cells partially through targeting GDF6 and FAPP2.

Reproduction (2020) 159 525-537
\end{abstract}

\section{Introduction}

Missed abortion (MA) is a special type of spontaneous abortion manifested as that intrauterine embryo stops growing and dies, but embryo and trophoblastic tissue are not spontaneously evacuated from uterine cavity in early pregnancy (Kafil \& Arain 2017). In the process of MA, placental tissue closely adheres to the uterine wall, causing difficulty in curettage (Xiao et al. 2019). It makes women confronting with the surgery and facing the risk of severe bleeding and complications associated with anesthesia. Previous studies have shown that there are multiple etiologic factors involved in the occurrence of MA, including genetic and uterine abnormalities, endocrine and immunological dysfunctions, infections, nutritional and environmental factors, psychogenetic factors, and endometriosis (Clifford et al. 1994, Hatasaka \& Gynecology 1994, Bulletti et al. 1996). However, the pathogenesis of the disease is currently unclear.

miRNAs are small, endogenous, noncoding singlestranded RNA molecules with 17-22 nucleotides. They degrade target mRNA or inhibit target mRNA translation by binding to $3^{\prime}$ untranslated regions ( $3^{\prime}$-UTR) of target mRNAs. They are highly conservative, tissue-specific and time-sequential and do not have an open reading frame. Up to 2018, the number of miRNAs annotated in miRBase has grown to 38,589 hairpin precursors and 48,860 mature miRNAs from 271 organisms (miRBase, release 22.1: October 2018). Almost onethird of the protein-coding genes are susceptible to be regulated by miRNAs post-transcriptionally (Lewis et al. 2005). By negatively regulating the expression of target genes, miRNAs are involved in the regulation of many central biological processes, such as cells development, proliferation, differentiation and apoptosis ( $\mathrm{Ni}$ et al. 2015), cancer proliferation and metastasis (Long et al. 2017). One miRNA can regulate many different genes and simultaneously a gene can be targeted by multiple miRNAs (Reinhart et al. 2000, Girard et al. 2008). Therefore, miRNA has been used to explore the pathogenesis of many diseases since been found. Recently, there had some reports demonstrate that abnormally expressed miRNAs are associated with pregnancy maintenance and pregnancy-specific diseases (Hromadnikova et al. 2012, Li et al. 2012, Zhu et al. 2016, Wang et al. 2015), indicating that miRNAs play an important role in pregnancy.

Our previous study indicates that miR-98 was differentially expressed in rat uteri during the periimplantation period and that down-regulation of miR-98 
in rat uterus during the receptive phase was linked to successful embryo implantation via targeting $B C l-x l$ (Xia et al. 2014). The implantation failure is one of major reasons for spontaneous abortion. And another study found that the expression of miR-98 was abnormal in deciduas of spontaneous miscarriage patients at 6-8 weeks (Wang et al. 2015). Therefore, we speculated that miR-98 may be associated with MA. In this study, we explore the relationship between miR-98 and MA occurrence and investigate the functional roles of miR-98 in trophoblast cell. Additionally, we also test the possible molecular mechanisms in which miR-98 is implicated.

\section{Materials and methods}

\section{Consent to participate}

All participants gave written informed consent and the study has been approved by Ethics Committee of Research Institute for Family Planning (2011-10).

\section{Tissue samples}

Two hundred and sixty samples of human MA placenta tissues and 396 samples of normal placenta tissues were collected from patients at Sixth Hospital of Shijiazhuang (Hebei, China). Both MA group samples and case group samples were taken before 12 weeks' gestation. Most of the possible factors led to spontaneous abortion were excluded, including the couple's chromosomal karyotype abnormality, anomaly of reproductive system, glucose level, T3/T4/TSH and sexual hormones level in maternal blood, immune antibodies (ANA/ACLA) test, virus infection (TORCH) test and the routine semen analysis from the patients' partner. Moreover, the patients who had genetic defects or hereditary diseases associated with spontaneous abortion in their family history were excluded.

Gestational age-matched normal gravidas who voluntarily chose induced abortion were served as controls. All samples were collected with informed consent of the patients. The tissues were frozen immediately in liquid nitrogen and stored at $-80^{\circ} \mathrm{C}$. This study has been approved by the ethics committee of National Research Institute for Family Planning (No.2011-06). We pair the placenta tissues by age, one case and one control. Hundred and seventy one pairs samples were sent to Beijing Longmaida Technology Co., Ltd. to make tissue microarrays, a technology that makes it possible to sample up to 1000 tissue samples on one glass slide. Analysis of qRTPCR was performed on 40 pairs samples that were 3 months pregnant from age groups $Y<25, Y 25-30, Y 30-35$ and $Y>35$.

\section{Cells culture and transfection}

HTR8/SVneo cells were gifted by Professor Yanling Wang from Institute of Zoology of Chinese Academy of Sciences (Beijing, China). The HTR8/SVneo cells were cultured in RPMI-1640 with $10 \%(\mathrm{v} / \mathrm{v})$ fetal bovine serum $(\mathrm{FBS}), 100 \mathrm{IU} / \mathrm{mL}$ penicillin and $100 \mathrm{IU} / \mathrm{mL}$ streptomycin in $5 \%(\mathrm{v} / \mathrm{v}) \mathrm{CO}_{2}$ atmosphere at $37^{\circ} \mathrm{C}$. MiR-98 mimic, miRNA mimic negative control, miR-98 inhibitor or miRNA inhibitor negative control (GenePharma,
China) was, respectively, transfected into the HTR8/SVneo cells through Lipofectamine 2000 Reagent (Invitrogen) according to the manufacturer's protocol. The final concentration miR-98 mimic, miRNA mimic negative control, miR-98 inhibitor and miRNA inhibitor negative control were $50 \mathrm{nM}$. Non-treated cells and cells treated with lipofectamine only were served as two additional controls. MiR-98 mimic is a double stranded microRNA molecule designed according to the sequence of mature miR-98, which is used to simulate the sequence of endogenous mature miRNA. MiR-98 inhibitor is sequencespecific and chemically modified to specifically target and knockdown individual miR-98 molecules. The designs of miRNA mimic negative control and miRNA inhibitor negative control are similar with miR-98 mimic and miR-98 inhibitor, respectively. However, either miRNA mimic negative control or miRNA inhibitor negative control is a random sequence based on C. elegans microRNA sequence that has been extensively tested in human cell lines and tissues and validated to produce no identifiable effects on known miRNA function.

\section{In situ hybridization of miR-98 with DIG-labeled LNA probe}

The tissue microarrays were treated with proteinase K (100 $\mu \mathrm{g} /$ $\mathrm{mL}$ ) for $15 \mathrm{~min}$ after deparaffinization by xylol and then refixed in $4 \%(\mathrm{w} / \mathrm{v})$ paraformaldehyde for $5 \mathrm{~min}$. After rinsing with phosphate buffer saline, the slides were prehybridized with hybridization buffer at $40^{\circ} \mathrm{C}$ for $1 \mathrm{~h}$ and then hybridized with digoxigenin (DIG)-labeled LNA-miR-98 probe (LNA-miR-98 sequences: $5^{\prime}$-DIG-aAcaaTaCAaCttaCtAcCtCa-3') overnight at $40^{\circ} \mathrm{C}$. On day 2 , the slides were washed in serial dilutions of saline sodium citrate solutions $(4 \times, 2 \times$ and $0.1 \times)$ and blocked with blocking reagent at room temperature for $30 \mathrm{~min}$, then incubated in blocking reagent with alkaline phosphatase (AP) labeled anti-DIG-antibody (Roche) overnight at $4{ }^{\circ} \mathrm{C}$. Staining was performed using BCIP/NBT Color Development Substrate (Promega). The sections were hybridized with DIGlabeled LNA-scrambled probe (LNA-scrambled sequences: 5'-caTtaAtgTcGgaCaaCtcAat-3') as negative control. Imaging was performed by iScanCoreo Au (Roche). Positive signals were quantitated by Image J software.

\section{Real-time PCR procedure to quantify miR-98 expression and target genes expression}

Total RNA was extracted by using TRIzol reagent and dissolved in diethylpyrocarbonate-treated water. The RNA concentration and purity were estimated by NanoDrop 2000 (ThermoFisher Scientific). The miR-98 expression was determined by TaqMan miRNA RT-Real Time PCR kit (ThermoFisher Scientific). U6snoRNA was used for normalization. Total mRNA was synthesized by $5 \times$ All-In-One RT MasterMix kit (Applied Biological Materials Inc). The mRNA level was amplified by $2 \times$ SYBR Green qPCR mix (Applied Biological Materials Inc) and the Real-Time PCR primers in Table 1. Gapdh gene was used for normalization. Relative miRNA/mRNA expression was calculated by efficiency-corrected $\Delta \Delta \mathrm{Ct}$ method. Each sample in each group was measured in triplicate, and the experiment was repeated for three times at least. 
Table 1 Primer sequences.

\begin{tabular}{llll}
\hline & \multicolumn{2}{c}{ Primer sequence $\left(5^{\prime} \rightarrow 3^{\prime}\right)$} \\
\cline { 2 - 3 } Gene name & Forward & Reverse & Application \\
\hline GDF6 3'-UTR & CTAGCTAGCGGTGACTGAAAGTGCAAAGAGG & ACGCGTCGACGCATATCATCCAGGAAAGGTGTAC & PCR \\
FAPP2 3'-UTR & CTAGCTAGCTCCTGTGTATGCGTGTCTG & ACGCGTCGACTAGCCTTTGGCATCTGGTC & PCR \\
mut-GDF6 3'-UTR & GAGTTTTTTTAAACTGTTTGCTTTATATAATACAAGT & TAAAGCAAACAGTTTAAAAAAAACTCTCCATTAGACAG & PCR \\
mut-FAPP2 3'-UTR & TGTCATTAAACAGCCCAATTGCTTTATGTGAAATTGGC & TAAAGCAATTGGGCTGTTAATGACATTCTAGTGAAAT & PCR \\
GDF6 & AATACCCATTCCCAGATAGTCAAA & CTAACTCTTCAAAGGGCCTACTC & qRT-PCR \\
FAPP2 & CCAAGGTGTGTGTGTGTTATTTC & ACGCGTCGACTAGCCTTTGGCATCTGGTC & qRT-PCR \\
\hline
\end{tabular}

\section{MTT assay}

Effect of miR-98 on viability of human trophoblasts cells was studied by MTT Assay. HTR8/SVneo cells were seeded in 96-well tissue culture plate (5000 cells per well) and incubated for $48 \mathrm{~h}$ after transfecting with miR-98 mimic, miRNA mimic negative control, miR-98 inhibitor and miRNA inhibitor negative control, respectively. The final concentration of miRNA mimic, inhibitor or corresponding controls was 50 nM. Non-treated cells and cells treated with lipofectamine only were served as two additional controls. Twenty microliters of MTT ( $5 \mathrm{mg} / \mathrm{mL}$; Promega Corporation) was added to each well and incubated at $37^{\circ} \mathrm{C}$. After $2 \mathrm{~h}$, the MTT was removed and $100 \mu \mathrm{L}$ of dimethyl sulfoxide was added to each well. The absorbance value of each well was read at $562 \mathrm{~nm}$ by SynergyMx microplate Reader (BioTek). Each sample was measured in triplicate, and all these assays were performed at least three times.

\section{Cell migration assay}

Effect of miR-98 on migration of human trophoblasts cells was studied by Transwell Assay. After 48 h of incubation, HTR8/ SVneo cells were transfected with miR-98 mimic, miRNA mimic negative control, miR-98 inhibitor and miRNA inhibitor negative control, respectively, in a 24-well plate. The final concentration of miRNA mimic, inhibitor or corresponding controls was $50 \mathrm{nM}$. Non-treated cells and cells treated with lipofectamine only served as two additional controls. Then the cells were trypsinized, washed and suspended in medium RPMI-1640 with $1 \%(\mathrm{v} / \mathrm{v})$ FBS. A total of $5 \times 10^{4}$ cells were seeded on the top of $8.0 \mathrm{~mm}$ pore membrane chamber (Corning CostarCorp.). To the bottom compartment, RPMI1640 with $5 \%(v / v)$ FBS was added as the chemotactic factor. The chamber was incubated for $12 \mathrm{~h}$. Then the non-migrated cells on the top of chamber were carefully removed with a cotton swab. Migrated cells on the bottom of chamber were fixed and stained with $0.1 \%(\mathrm{w} / \mathrm{v})$ crystal violet solution. The cell migration was quantified by the number of cells passing through the pores from five different fields per sample. All experiments were performed at least three times.

\section{EdU assay}

The proliferation of HTR8/SVneo cells was determined by Cell-Light ${ }^{\mathrm{TM}}$ EdUApollo®567 In Vitro Imaging kit (Ribobio) according to the manufacturer's instructions. Cells were transfected with the miR-98 mimic, miRNA mimic negative control, miR-98 inhibitor and miRNA inhibitor negative control, respectively, in a 24-well plate. The final concentration of miRNA mimic, inhibitor or corresponding controls was $50 \mathrm{nM}$. Non-treated cells and cells treated with lipofectamine only were served as two additional controls. $\mathrm{EdU}$ is a thymidine analog which can label cells when DNA replicating (Salic \& Mitchison 2008). Cells were cultured in 24-well plates with $1 \times 105$ cells/well and exposed to $50 \mu \mathrm{M}$ $\mathrm{EdU}$ for $2 \mathrm{~h}$ at $37^{\circ} \mathrm{C}$, then fixed in $4 \%(\mathrm{w} / \mathrm{v})$ formaldehyde for $30 \mathrm{~min}$ at room temperature and permeabilized in $0.5 \%(\mathrm{v} / \mathrm{v})$ Triton X-100 for $10 \mathrm{~min}$. Subsequently, the cells were washed with PBS, and each well was incubated with $200 \mu \mathrm{L} 1 \times$ Apollo reaction cocktail for $30 \mathrm{~min}$. DNA was stained with $200 \mu \mathrm{L}$ $1 \times$ Hoechst33342 for $30 \mathrm{~min}$ and imaged under Confocal Microscope (LSM 710; Zeiss). This experiment was repeated for at least three times.

\section{Plasmid construction}

The GDF6 3'-UTR and FAPP2 3'-UTR sequences were amplified by PCR from human genomic DNA using the primers in Table 1. After being double digested with Sall and Nhel, the PCR product was cloned into pmirGLO vector (Invitrogen). The GDF6 3'-UTR-mutant and FAPP2 3'-UTR-mutant vector were obtained following Fast Mutagenesis System (TransGen Biotech) using the primers in Table 1. Scramble siRNA control, GDF6 siRNA and FAPP2 siRNA were synthesized by GenePharma.

\section{Dual-luciferase activity assay}

The HTR8/SVneo cells seeded in a 48-well plate were treated with recombinant plasmid and miR-98 mimic, miRNA mimic negative control, miR-98 inhibitor and miRNA inhibitor negative control, respectively, through co-transfection with Lipofectamine 2000 Reagent, according to the manufacturer's protocol (siRNA Plasmid Co-Transfection Protocol with Lipofectamine 2000, Invitrogen). The final concentration of miRNA mimic, inhibitor or corresponding controls was $50 \mathrm{nM}$. The concentration of plasmid DNA was $0.5 \mathrm{ng} / \mu \mathrm{L}$. After $48 \mathrm{~h}$ of transfection, cells were harvested and lysed. The luciferase reporter activities were measured using Dual-Glo ${ }^{\circledR}$ Luciferase Assay System (Promega). Each sample was measured in triplicate, and all experiments were repeated for at least three times.

\section{Western blotting analysis}

Cell protein lysates were collected in RIPA Lysis Buffer. Protein concentration was measured by Easy II Protein Quantitative kit 
(BCA) (TransGen). Sixty microgram of total protein was separated on $12 \%(\mathrm{w} / \mathrm{v})$ acrylamide gel by electrophoresis and transferred to polyvinylidene fluoride membrane (Amersham Pharmacia Biotech), probed with antibodies against human GDF6 (1:250, Immunoway), FAPP2 (1:250, Proteintech) and anti- $\beta$ actin polyclonal antibody (1:250, Santa Cruz Biotechnology Inc.), respectively, overnight at $4^{\circ} \mathrm{C}$. Horseradish peroxidase (HPR) conjugated goat anti-rabbit IgG (1:10,000, Biodragon) were used to detect primary antibodies. Chemiluminescence reaction was visualized on ECL kit (Millipore). The experiment has been repeated for at least three times.

\section{Immunohistochemistry}

The tissue microarrays $(6 \mu \mathrm{m})$ were deparaffinized in xylene and rehydrated in descending ethanol series. Antigen retrieval was accomplished through autoclave boiling the slide in $10 \mathrm{mM}$ sodium citrate buffer. Then the slide was incubated with rabbit anti-GDF6 polyclonal antibody (1:100, Immunoway) and rabbit anti-FAPP2 polyclonal antibody (1:100, Proteintech), respectively, then incubated with HRP-conjugated goat anti-rabbit IgG (1:500, BiodragonTechnology Co.). The antibody stains were developed through diaminobenzidine (ZsbioTechnology Co.) and nuclei were stained with hematoxylin (Sigma-Aldrich). The slide was incubated with fetal bovine serum as negative control. Digital images were obtained from slice scanning device NanoZoomer S210 (Hamamatsu). Positive signal was quantitatively analyzed through ImageJ software with $\mathrm{H}$-score grading system (BudwitNovotny et al. 1986). Random ten-pair samples in each age group $(Y<25, Y 25-30, Y 30-35, Y>35)$ were selected for optical density quantitative analysis.

\section{Statistical analysis}

The GraphpadPrism 7.0 software was applied for statistical analyses. Data are presented as mean \pm S.E.M. from at least three independent experiments. Two groups comparisons were performed using $t$-test and multiple groups comparisons

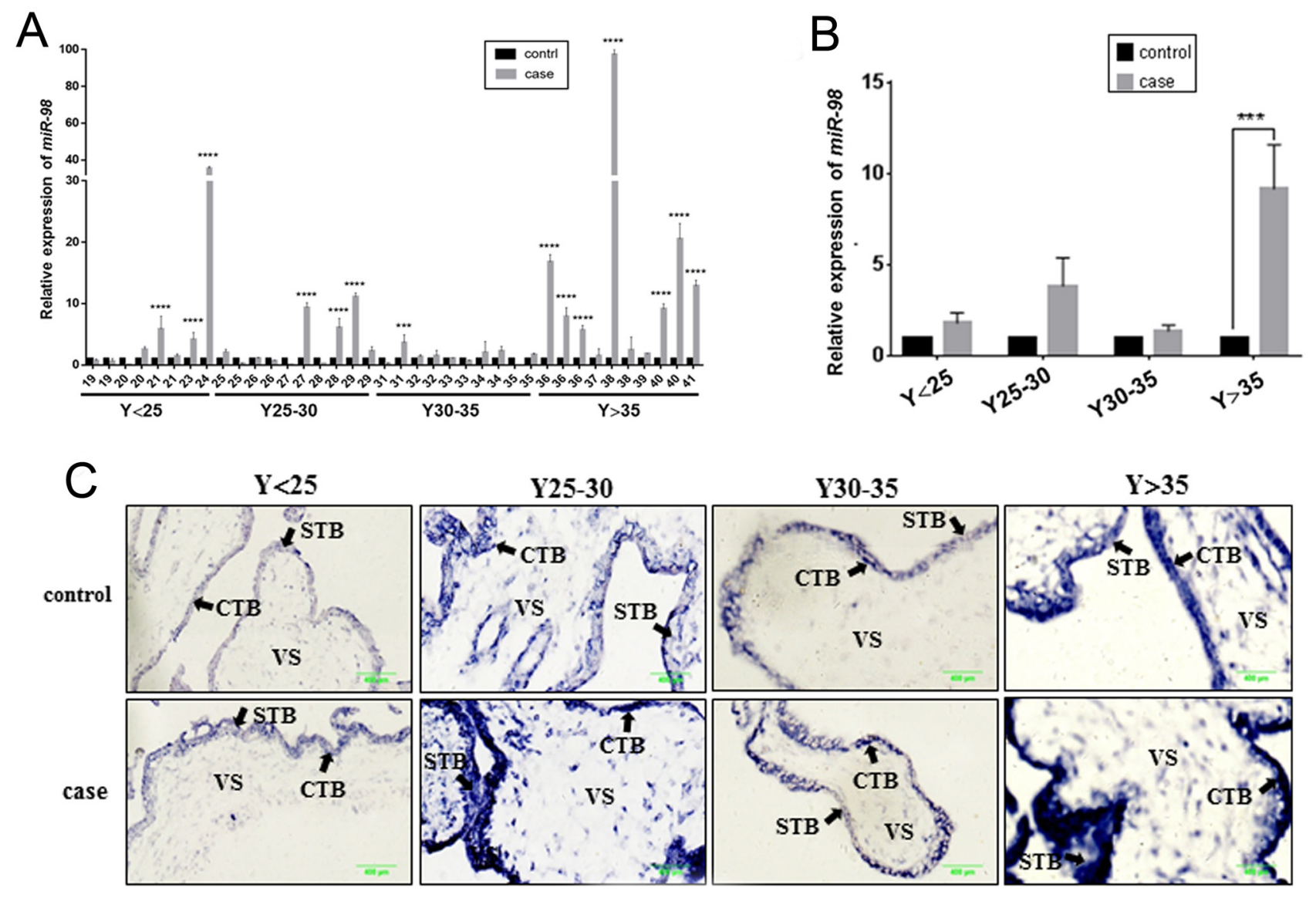

Figure 1 qRT-PCR and ISH analysis of missed abortion placental tissues and normal tissues. (A) The relative expression of miR-98 in individual sample was detected by TaqMan miRNA RT-Real Time PCR. U6 snoRNA was used for normalization. (B) The relative expression of miR-98 in individual sample was grouped in 25 years old ( $\mathrm{Y}<25$ ), 25-30 years old ( $\mathrm{Y} 25-30)$, 30-35 years old ( $\mathrm{Y} 30-35)$, over 35 years old ( $\mathrm{Y}>35$ ). (C) ISH shows that miR-98 was expressed both in MA sample (case group) and normal villi tissues (control group) at early pregnancy and mainly distributed in villi syncytiotrophoblast, cytotrophoblast and villous stroma. Differential expression of miR-98 in MA placental tissues between different age groups compared to normal tissues. Strong miR-98 staining signal (blue purple) in MA placental tissues and weak signal in normal placenta tissues. ISH: in situ hybridization; CTB: cytotrophoblast; STB: syncytiotrophoblast; VS: villous stroma. The scale bar is $400 \mu \mathrm{m}$. *** $P<0.001, * * * * P<0.0001$. 
using one-way ANOVA. Differences were considered significantly at $P<0.05$.

\section{Results}

\section{miR-98 was up-regulated in the placental villi from MA patients}

In order to explore the role of miR-98 in MA, the expression patterns of miR-98 in the placental villi from MA patients and matching normal placental villi were analyzed. Because advanced age is a risk factor for missed abortion (Zhang et al. 2011), the placental villi tissues were divided into four groups according to the maternal age, including under 25 years old $(\mathrm{Y}<25)$, 25-30 years old (Y25-30), 30-35 years old (Y30-35), over 35 years old $(Y>35)$.

qRT-PCR was used to determine the miR-98 level in the placental villi from MA patients (Fig. 1A and B). We firstly analyzed the miR-98 expression level in a single pair of case and control samples (Fig. 1A). In 38 pairs samples, miR-98 level was up-regulated in 26 pairs samples and down-regulated in 12 pairs samples. However, only 14 pairs of up-regulation of miR-98 levels showed statistically significant differences (at least $P<0.05$ ). We further analyzed the percentage of miR-98 up-regulation in different age groups. The results showed that the percentage of up-regulation of miR-98 expression in the placental villi from MA patients was $37.50 \%$ (3/8 pair samples) in $Y<25$ group, $30.00 \%$ (3/10 pair samples) in Y25-30 group, $10 \%$ (1/10 pair samples) in Y30-35 group and $70 \%$ (7/10 pair samples) in $Y>35$ group. The

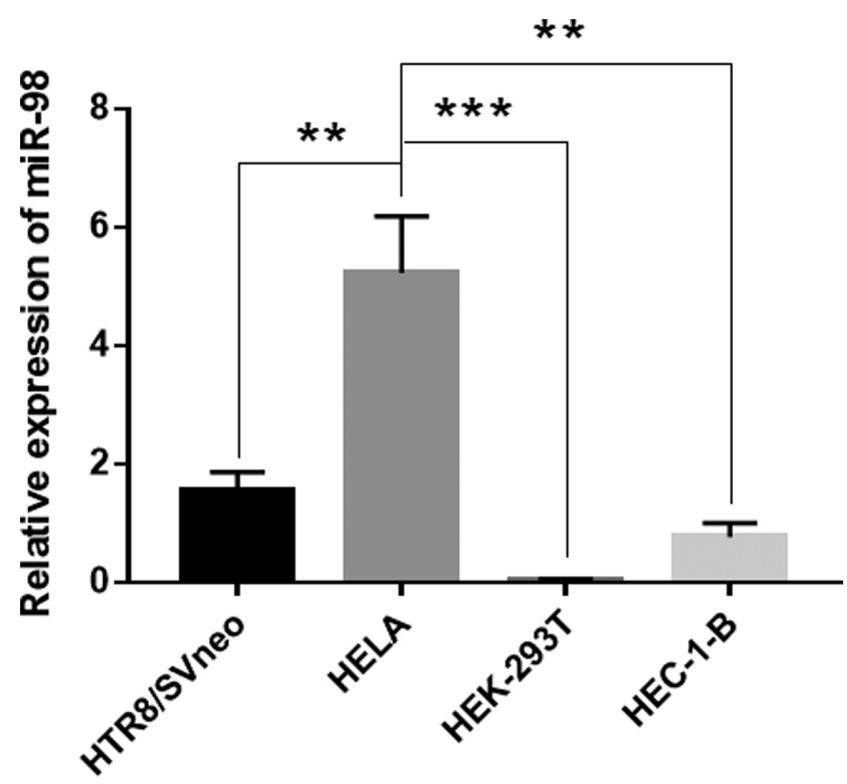

Figure 2 miR-98 expression level in different cell lines. The relative expression of miR-98 in HTR8/SVneo, HELA, HEK-293T and HEC-1-B was detected by TaqMan miRNA RT-Real Time PCR. The $y$-axis displays the relative $\log 2$ ratio of miR-98 normalized by U6. ${ }^{* *} P<0.01, * * * P<0.001$. average level of miR-98 in placental villi from MA patients was higher than that in control group in $Y>35$ group $(P<0.001$; Fig. 1B).

In situ hybridization (ISH) was performed on placental villi tissues to clarify the distribution of miR-98 (Fig. 1C). It demonstrated that miR-98 was expressed both in MA samples and normal villi tissues at early pregnancy and mainly distributed in villi syncytiotrophoblast, cytotrophoblast and villous stroma. In different age groups, miR-98 staining signals were strong in MA placental tissues and weak in normal placenta tissues.
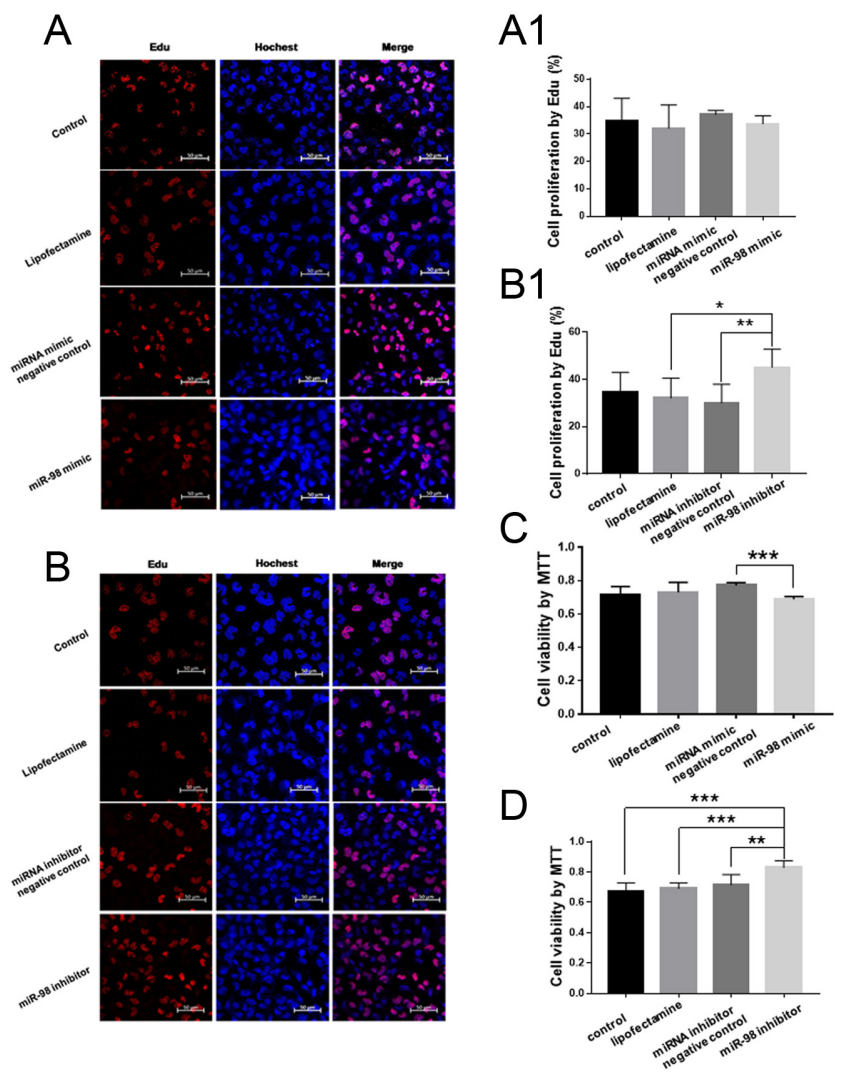

Figure 3 The effects of miR-98 on proliferation and viability. (A and B) Cells proliferation was determined by Edu assay. Red represents the proliferative cells and blue indicates cell nuclei. HTR8/SVneo cells transfected with miRNA mimic negative control, miR-98 mimic, miRNA inhibitor negative control and miR-98 inhibitor, respectively, were incubated for $48 \mathrm{~h}$. The final concentration of miRNA mimic negative control, miR-98 mimic, miRNA inhibitor negative control and miR-98 inhibitor was $50 \mathrm{nM}$. Non-treated cells and cells treated with lipofectamine only were served as two additional controls. The scale bar is $50 \mu \mathrm{m}$. (A1 and B1) The cell proliferation was quantified by counting the amount of Edu-positive cells vs total cells from five different fields per sample in randomly selection. (C and D) HTR8/ SVneo cells were transfected with the miRNA mimic negative control, miR-98 mimic, miRNA inhibitor negative control and miR-98 inhibitor, respectively. The final concentration was $50 \mathrm{nM}$. Using non-treated cells and cells treated with lipofectamine only for additional controls. After $48 \mathrm{~h}$, cells viability was determined by MTT assay. The $y$-axis displays the OD value at A570 nm. ${ }^{*} P<0.05$, ${ }^{* *} P<0.01,{ }^{* * *} P<0.001$. 

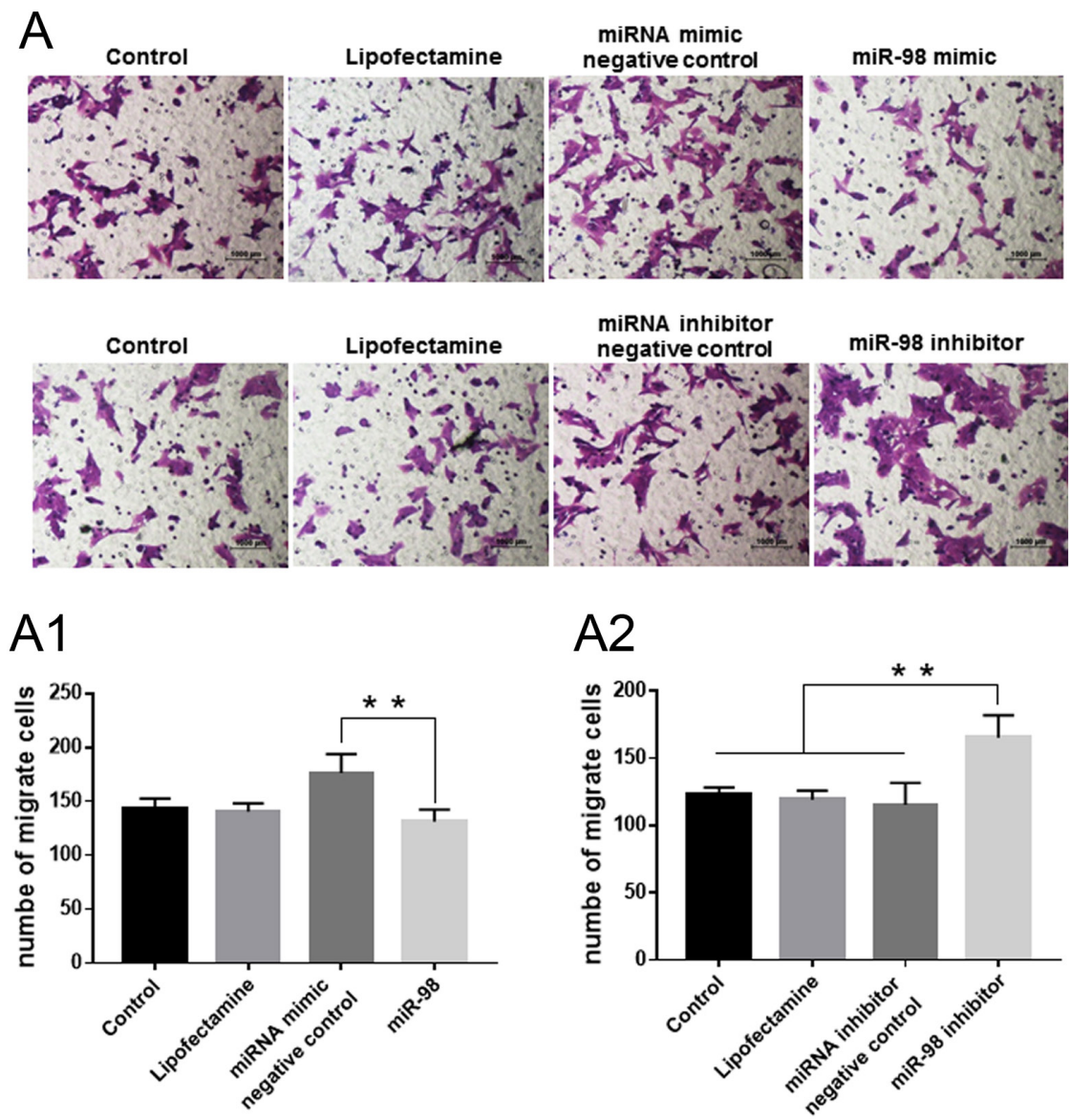

Figure 4 The effects of $m i R-98$ on migration of trophoblastic cells. (A) The migration of HTR8/ SVneo cells were detected by transwell assay. HTR8/SVneo cells were transfected with miRNA mimic negative control, miR-98 mimic, miRNA inhibitor negative control and miR-98 inhibitor, respectively. The final concentration was $50 \mathrm{nM}$. Non-treated cells and cells treated with lipofectamine only were served as two additional controls. After $48 \mathrm{~h}$, cells from each group were transferred separately to transwell chambers at density of $0.5 \times 10^{6}$ cell $\mathrm{s} / \mathrm{mL}$. After incubation for $16 \mathrm{~h}$, the cells on top of the transwell chamber were removed and the cells on the bottoms were stained with crystal violet. The scale bar is $1000 \mu \mathrm{m}$. (A1 and A2) The cell migration was quantified by counting the amount of cells passing through the membrane from five different fields per sample at $100 \times$ in randomly selection. ${ }^{* *} P<0.01$.

\section{Overexpression of miR-98 suppresses the proliferation of HTR8/Svneo Cells in vitro}

To study the possible effect of miR-98 in the pathological process of placenta, HTR8/Svneo cells have been selected to be the in vitro cell lines model, as it developed from human placenta villus trophoblast cells and expressed miR-98 modestly according to qRT-PCR result (Fig. 2).

Edu assay was applied to observe the effect of miR-98 on proliferation of HTR8/Svneo cells (Fig. 3A and $B$ ). The relative proliferation rates in HTR8/SVneo cells transfected with miR-98 inhibitor was significantly higher than that in the miRNA inhibitor negative control group and cells treated with lipofectamine only $(P<0.05, P<0.01$; Fig. 3B1). Compared with miRNA mimic negative control, the relative proliferation rates in miR-98 mimic had downward trends, but no statistically significant differences.

\section{Overexpression of miR-98 suppresses the viability of HTR8/Svneo cells in vitro}

MTT assay was used to further assess the effect of miR-98 on the viability of HTR8/Svneo cells (Fig. 3C and D). The viability in cells transfected with miR-98 mimic was obviously weaker than that miRNA mimic negative control group $(P<0.001)$. MiR-98 inhibitor significantly strengthened cell viability as compared with miRNA inhibitor negative control group, non-treated cells and cells treated with lipofectamine only $(P<0.01$ and $P<0.001)$.

\section{Overexpression of miR-98 inhibits the migration of HTR8/Svneo cells in vitro}

The influence of miR-98 on HTR8/Svneo cells migration was detected by transwell assay (Fig. 4A). The results displayed that the migration of HTR8/Svneo cells have no difference among non-treated cells, cells treated with lipofectamine-only and miRNA mimic negative control or miRNA inhibitor negative control. The migration of HTR8/Svneo cells transfected with miR-98 mimic was significantly lower than the miRNA mimic negative control group $(P<0.01$; Fig. 4A1). Otherwise, the migration of HTR8/Svneo cells was enhanced in miR-98 inhibitor group $(P<0.01$; Fig. 4A2). These findings suggest that miR-98 suppressed the migration of HTR8/Svneo cells.

\section{miR-98 negatively regulates GDF6 and FAPP2 expression by binding to their $3^{\prime}$-UTR}

To elucidate the molecular mechanisms of miR98 effects in placenta development, computational 
A

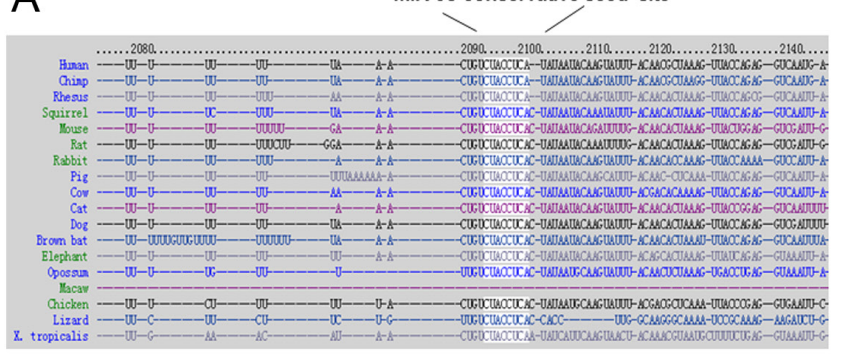

B

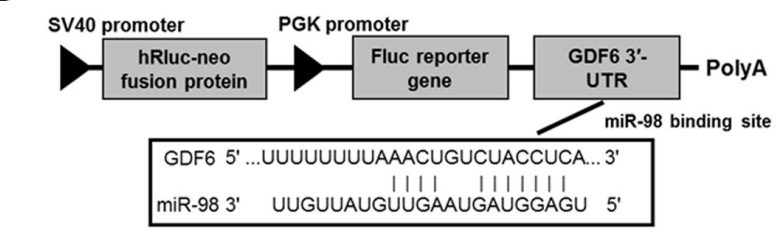

C

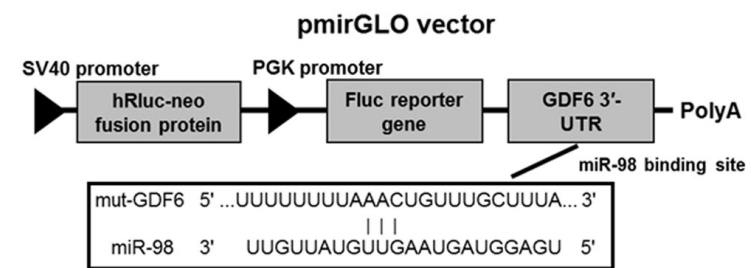

B1

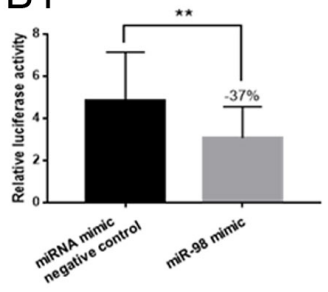

C1

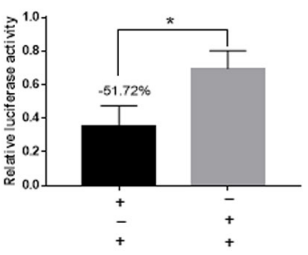

D1

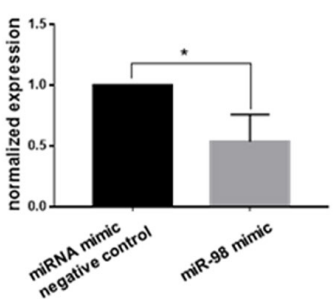

E1

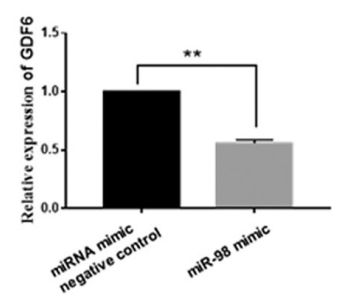

B2

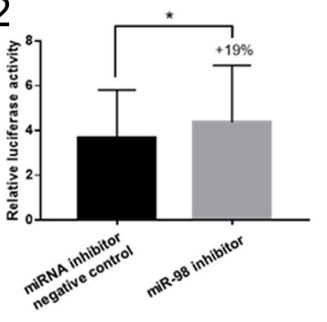

D

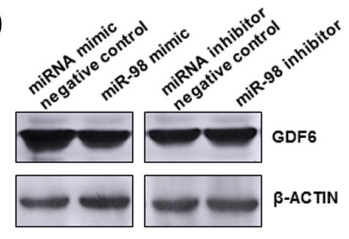

GDF6-pGLO $=$

D2

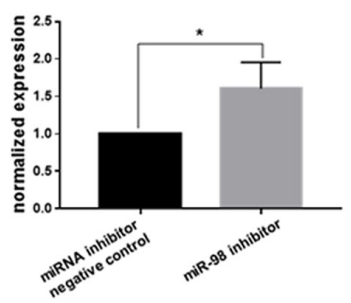

E2

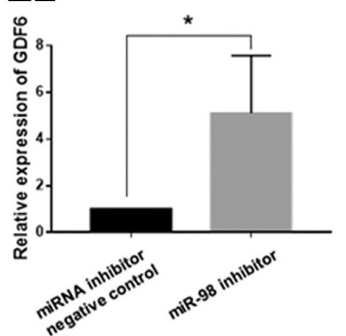

Figure 5 The prediction and confirmation of the miR-98 target gene GDF6. (A) miR-98 has a conservative binding site in GDF6 3'-UTR regions between different species. (B) Schematic diagram for constructing miR-98 binding site into pmirGLO vector. (B1 and B2) Confirmation of miR-98 target gene GDF6 through transfecting HTR8/Svneo cells with miRNA mimic negative control, miR-98 mimic, miRNA inhibitor negative control and miR-98 inhibitor, respectively, for dual-luciferase assay. The final concentration of miRNA mimic, inhibitor or corresponding controls was 50 $\mathrm{nM}$ and the concentration of plasmid DNA was $0.5 \mathrm{ng} / \mu \mathrm{L}$. (C) Schematic diagram for constructing miR-98 mutant binding site into pmirGLO vector. (C1) Mutation analysis of the miR-98 binding site. (D) GDF6 protein level in HTR8/Svneo cells treated with miR-98 mimic and miR-98 inhibitor. The expression of $\beta$-ACTIN served as an internal control. The black histogram represents the optical densities of signals quantified by ImageJ and normalized with $\beta$-ACTIN intensity. (E1 and E2) GDF6 expression level in HTR8/Svneo cells treated with miR-98 mimic and inhibitor. The expression of GDF6 mRNA in HTR8/Svneo cells were detected by qRT-PCR. Gapdh serves as an internal reference. The y-axis displays the relative log2 ratio of GDF6 normalized by Gapdh. ${ }^{*} P<0.05,{ }^{* *} P<0.01$.

approaches were applied to predicted putative genes for miR-98. MiRWalk and Targetscan are two online databases on predicting and publishing miRNAs. By overlapping data between the two databases, we chose GDF6 and FAPP2 to study, since GDF6 related to embryonic development and FAPP2 abundantly expressed in placenta (Fagerberg et al. 2014, Hwang et al. 2015, Wei et al. 2016). The responsive sites in 3'-UTR of GDF6 and FAPP2 are both highly conserved among different species (Figs $5 \mathrm{~A}$ and $6 \mathrm{~A}$ ). The pairing types of GDF6 and FAPP2 responsive element in $3^{\prime}-$ UTR to the seed region of miR-98 are both 8 mer and have different complementarity degrees with miR-98 in non-responsive region of $3^{\prime}$-UTR (Figs 5B and 6B).

To investigate whether GDF6 or FAPP2 is the target gene of miR-98, their 3'-UTR fragment containing responsive sites was respectively cloned into pmirGLO control vectors, which have luciferase reporter genes. Recombinant plasmids were co-transfected into HTR8/ SVneo cells with miR-98 mimic, miRNA mimic negative control, miR-98 inhibitor and miRNA inhibitor negative control, respectively. Detecting by dual-luciferase assay, the luciferase activity was significantly reduced in miR-98 mimic group compared to miRNA mimic 
A

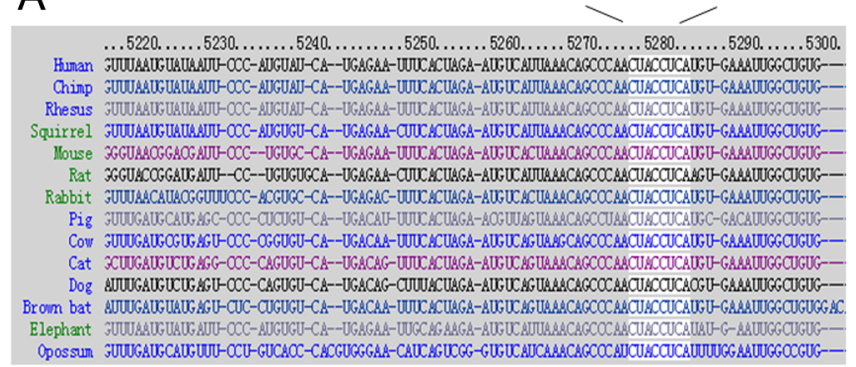

B

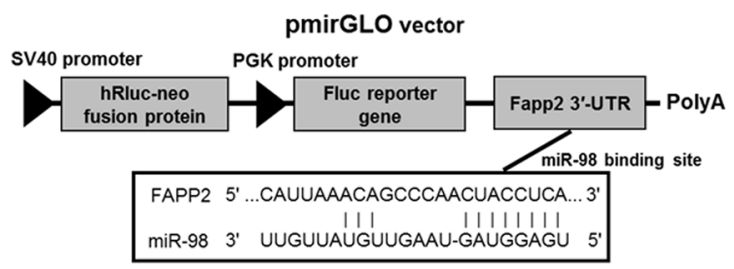

C

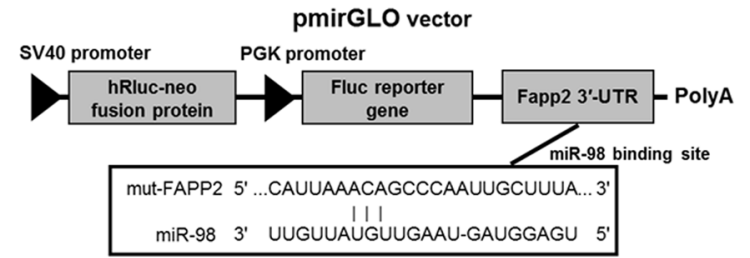

B1

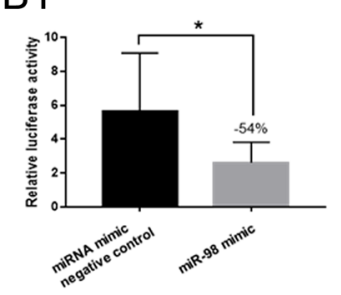

B2
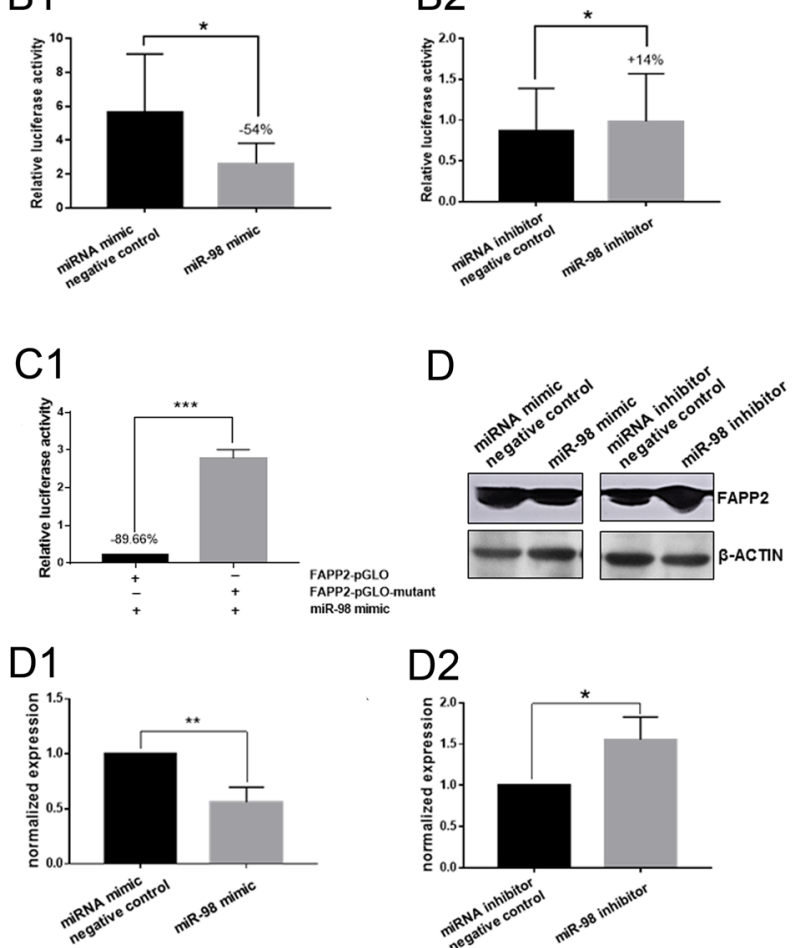

D2

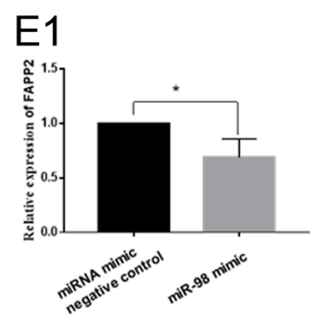

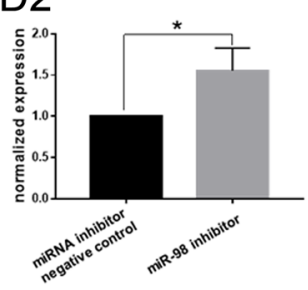

E2

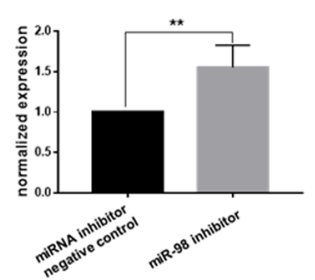

Figure 6 The prediction and confirmation of the miR-98 target gene FAPP2. (A) miR-98 conservative binding sites in FAPP2 3'-UTR region between different species. (B) Schematic diagram for constructing miR-98 binding site into pmirGLO vector. (B1 and B2) Confirmation of miR-98 target gene FAPP2 through transfecting HTR8/Svneo cells with miRNA mimic negative control, miR-98 mimic, miRNA inhibitor negative control and miR-98 inhibitor, respectively, for dual-luciferase assay. The final concentration of miRNA mimic, inhibitor or corresponding controls was 50 $\mathrm{nM}$ and the concentration of plasmid DNA was $0.5 \mathrm{ng} / \mu \mathrm{L}$. (C) Schematic diagram for constructing miR-98 mutant binding site into pmirGLO vector. (C1) Mutation analysis of the miR-98 binding site. (D) FAPP2 protein level in HTR8/Svneo cells treated with miR-98 mimic and miR-98 inhibitor. The expression of $\beta$-ACTIN served as an internal control. The black histogram represents the optical densities of the signals quantified by ImageJ and normalized with $\beta$-ACTIN intensity. (E1 and E2) FAPP2 expression level in HTR8/Svneo cells treated with miR-98 mimic and inhibitor. The expression of FAPP2 in HTR8/Svneo cells were detected by qRT-PCR. Gapdh serves as an internal reference. The y-axis displays the relative $\log 2$ ratio of FAPP2 normalized by Gapdh. ${ }^{*} P<0.05,{ }^{* *} P<0.01,{ }^{* * *} P<0.001$.

negative control group (GDF6-37\%, $P<0.01$, Fig. 5B1; FAPP2-54\%, $P<0.05$, Fig. 6B1). Conversely, the luciferase activity was obviously increased by miR-98 inhibitor treatment $(\mathrm{CDF} 6+19 \%, P<0.05$, Fig. 5B2; FAPP $2+14 \%, P<0.05$, Fig. $6 \mathrm{~B} 2)$. To further validate the targeting relationship between GDF6, FAPP2 and miR-98, mutation of responsive sites in recombinant plasmids was constructed for dual luciferase assay. Unmutated recombinant plasmids were utilized to be control. Under the influence of miR-98 mimic, the luciferase activity of the control group was significantly lower than that of the mutation group (CDF6-51.72\%,
$P<0.05$, Fig. 5C1; FAPP2-89.66\%, $P<0.001$, Fig. 6C1). These data suggested that miR-98 could down-regulate GDF6 and FAPP2 expression through binding to their responsive sites at the $3^{\prime}$-UTR. Therefore, it is better understood that both GDF6 and FAPP2 are direct target genes of miR-98.

To confirm whether miR-98 could regulate endogenous GDF6 and FAPP2 expression, HTR8/ SVneo was transfected transiently with $m i R-98$ mimic and miR-98 inhibitor, respectively. The Western blot results showed that the GDF6 protein was significantly decreased after miR-98 mimic transfected and greatly 
A

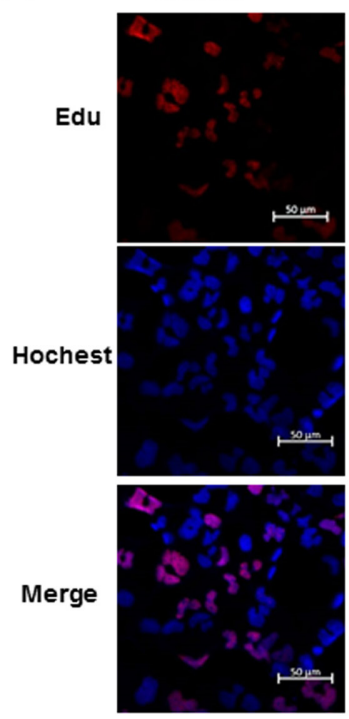

miR-98 inhibitor
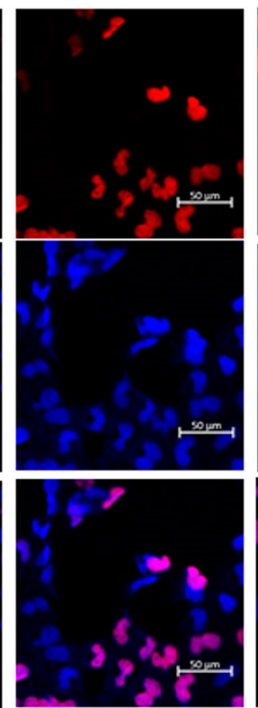
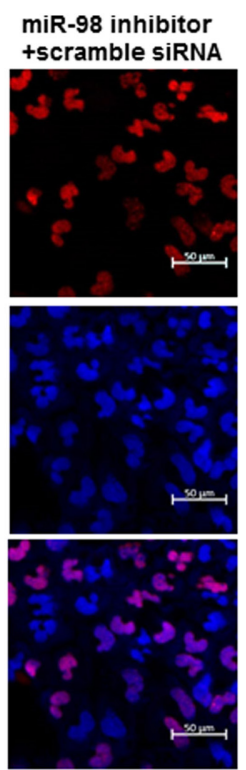

miR-98 inhibitor +GDF6-siRNA
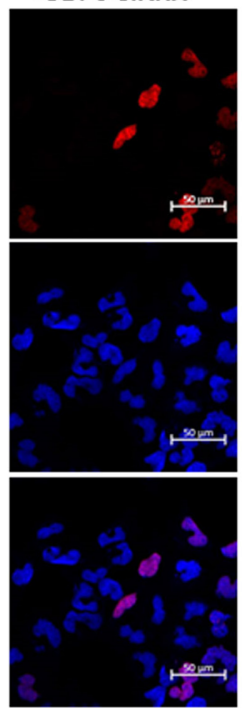

B control miR-98 inhibitor +scramble siRNA
miR-98 inhibitor

miR-98 inhibitor +GDF6-siRNA
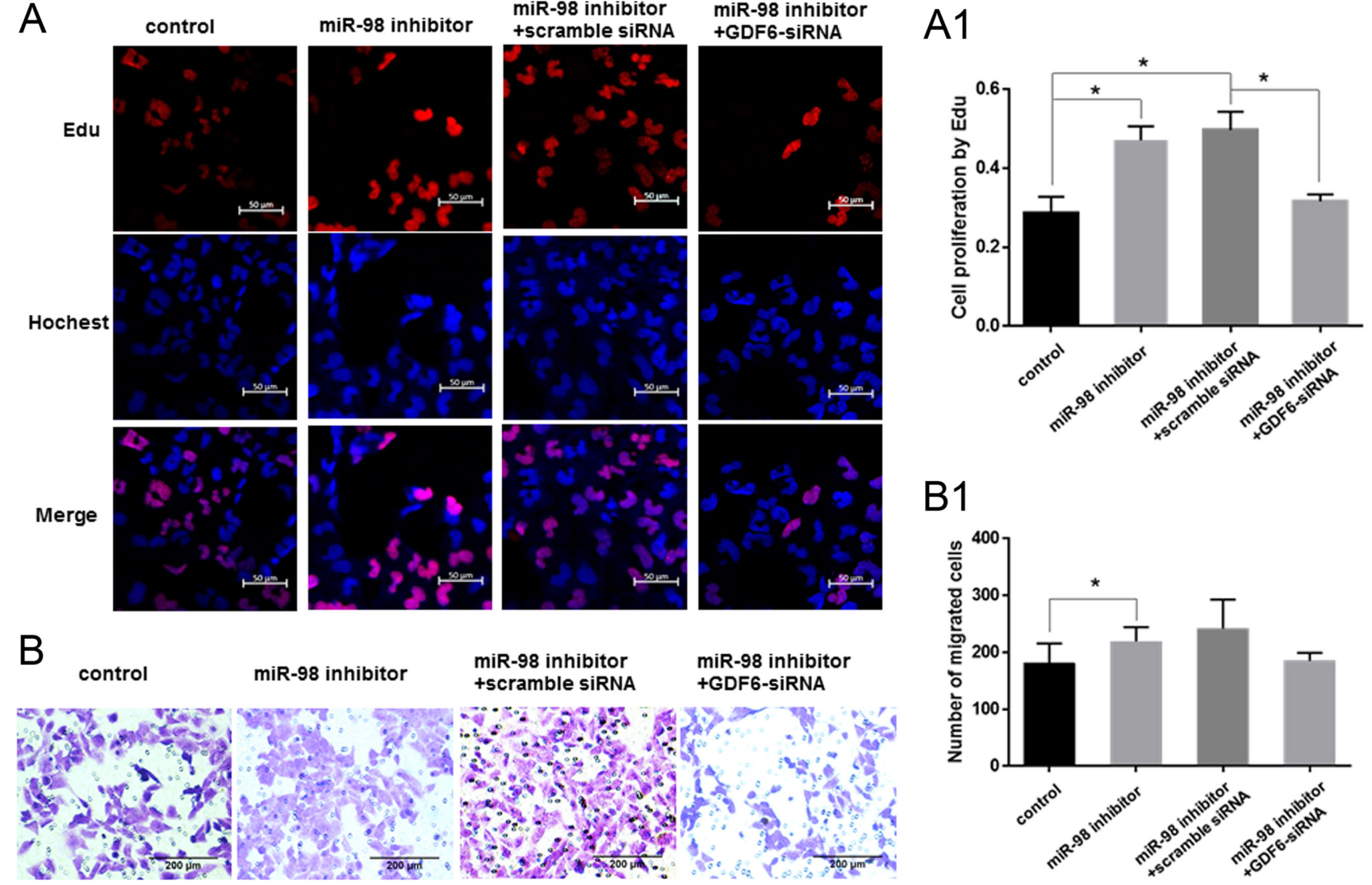

B1

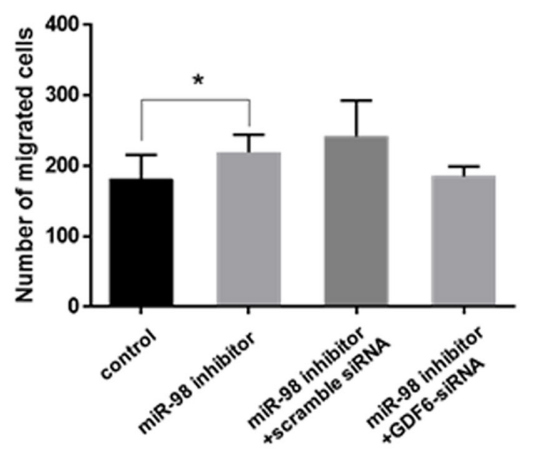

Figure 7 Knockdown of GDF6 attenuates the down-regulation of miR-98 mediated enhancement of cell growth. GDF6 siRNA and scramble siRNA were transfected into HTR8/Svneo cells with miR-98 inhibitor, respectively. (A) Cell proliferation was detected by Edu assay. The photographs are shown at $200 \times$ and the scale bar is $50 \mu \mathrm{m}$. (B) Cell migration was determined by transwell assay. The photographs are shown at 200x. The scale bar is $200 \mu \mathrm{m} .{ }^{*} P<0.05$.

increased in the miR-98 inhibitor transfected group compared to negative control group $(P<0.05$, Fig. 5D1; $P<0.05$, Fig. 5D2, Supplementary Fig. 1, see section on supplementary materials given at the end of this article). In addition, as the result of qRT-PCR showed, the level of GDF6 mRNA was significantly down-regulated by miR-98 mimic and up-regulated by miR-98 inhibitor in HTR8/SVneo cells $(P<0.01$, Fig. 5E1; $P<0.05$, Fig. 5E2). Similar results were obtained when FAPP2 expression levels were detected by Western blot and qRT-PCR $(P<0.05, P<0.01$, Fig. 6D1 and D2; $P<0.05, P<0.01$, Fig. $6 \mathrm{E} 1$ and E2).

\section{miR-98 effect HTR8/SVneo cells proliferation and migration by targeting GDF6 and FAPP2}

To further study the possible functions of miR-98 mediating GDF6 and FAPP2, rescue experiments were conducted on HTR8/SVneo cells. GDF6 siRNA and miR98 inhibitor were co-transfected into HTR8/SV neo cells. As the results show, proliferation capacity of the cells co-transfected by GDF6 siRNA and miR-98 inhibitor was significantly decreased compared to that co-transfected by scramble siRNA and miR-98 inhibitor $(P<0.05$,
Fig. 7A1). Similar results of proliferation and migration were obtained when the cell was co-transfected with FAPP2 siRNA and miR-98 inhibitor $(P<0.05$, Fig. 8A1 and B1). These findings implied that miR-98 mediated enhancement on cell proliferation was down-regulated by knockdown of GDF6 and FAPP2. They also suggest that miR-98 could reduce cell proliferation and migration through suppression of GDF6 and FAPP2 expression.

\section{Downregulation of GDF6 and FAPP2 expression in the placental villi from MA patients}

To further explore the expression profiles of miR-98 target genes GDF6 and FAPP2, the expression and distribution of GDF6 and FAPP2 in human placental tissues with MA were determined by immunohistochemistry. Both GDF6 and FAPP2 were widely detected in MA tissues and normal tissues (Fig. 9A). They were mainly localized in villi syncytiotrophoblast, cytotrophoblast and villous stroma. The results reflected that the GDF6 expression in placental villi of MA patients at each age was slightly less than that of control, but there was no statistical difference (Fig. 9A1). The expression of FAPP2 in MA patients has great reduce in $Y<25$ group and $Y 30-35$ 

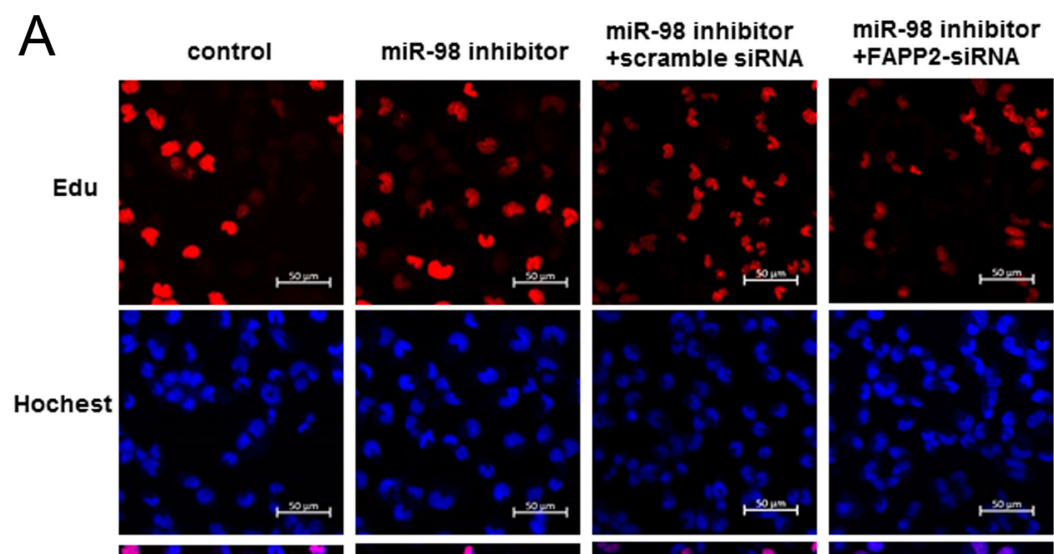

\section{A1}
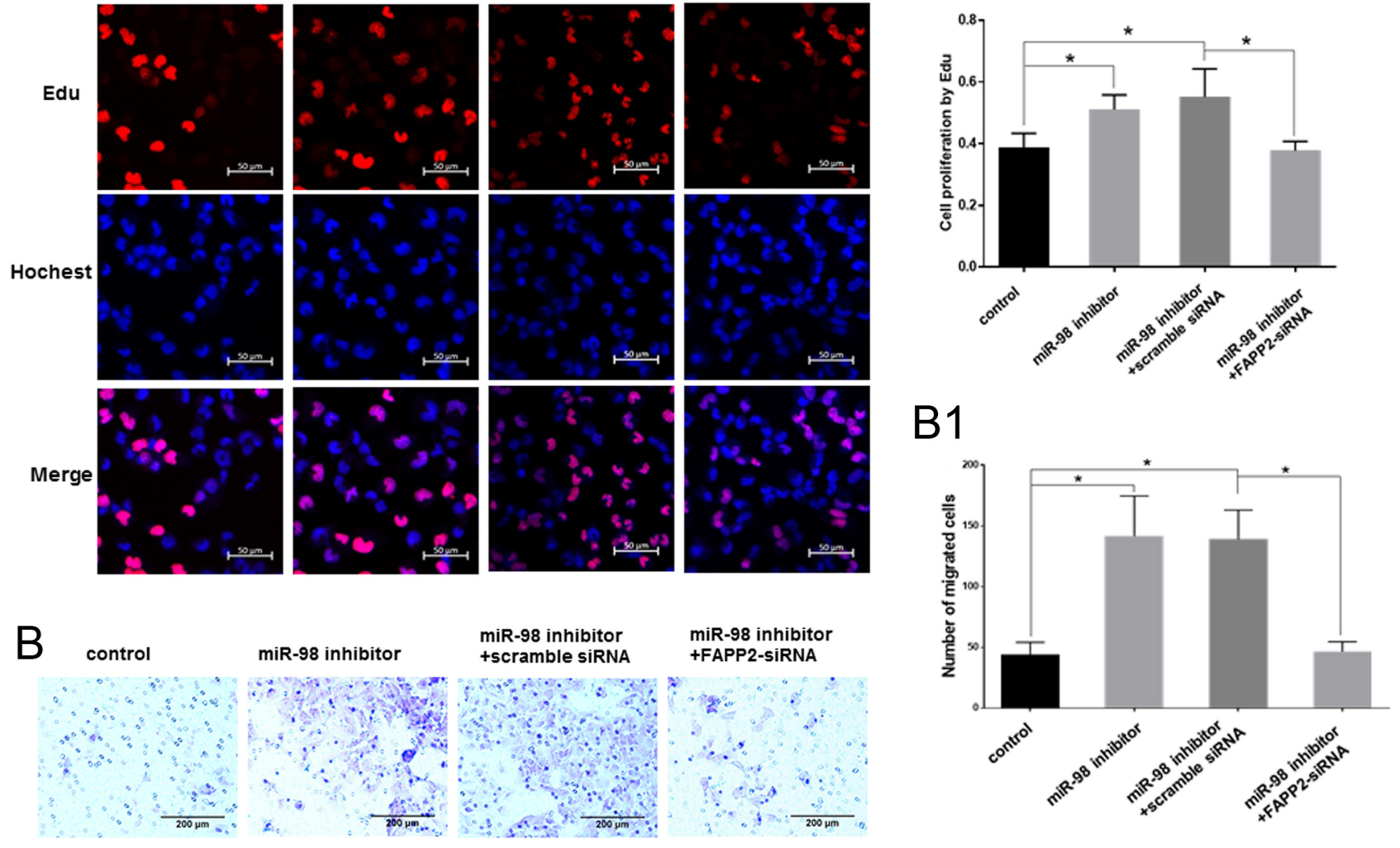

Figure 8 Knockdown of FAPP2 attenuates the down-regulation of miR-98 mediated enhancement of cell growth. FAPP2 siRNA and scramble siRNA were transfected into HTR8/Svneo cells with the miR-98 inhibitor, respectively. (A) Cell proliferation was detected by Edu assay. The photographs are shown at $200 \times$. The scale bar is $50 \mu \mathrm{m}$. (B) Cell migration was determined by transwell assay. The photographs are shown at $200 \times$. The scale bar is $200 \mu \mathrm{m} .{ }^{*} P<0.05$.

group $(P<0.01, P<0.05$, Fig. 9A2). The expression trend of GDF6 and FAPP2 were opposite with miR-98 in MA placental tissues, indicating that $m i R-98$ may be involved in the occurrence of MA by negatively regulating GDF6 and FAPP2.

\section{Discussion}

In this study, we found that miR-98 is significantly increased in MA placental villi tissues compared to normal tissues, suggesting that up-regulation of miR-98 may be associated with the occurrence of MA. As far as we know, our research is the first study to examine the relationship between miR-98 and the occurrence of MA.

Our findings demonstrate that miR-98 was up-regulated in the placental villi from MA patients. We further explored the biological functions of miR98 in MA and found that overexpression of miR-98 in HTR8/SVneo trophoblast cells in vitro inhibited cells proliferation, activity and migration. Interestingly, it has been found that miR-98 prevents the proliferation, migration and invasion of cells in many cancers (Ni et al. 2015, Fu et al. 2018). The role of miR-98 in human trophoblastic cells was coincident with that in cancers. Consequently, all the facts show that miR98 is a cell growth suppressor. Proliferation, migration and invasion of trophoblastic cells into the maternal endometrium are essential steps in embryo implantation and placentation (Cohen et al. 2010). Up-regulation of miR-98 in MA placental villi tissues may result in a shallow invasion of the trophoblast into the decidua and maternal vessels and affect the fetal nutrition supply and then may cause embryo death and the occurrence of MA.

To clarify the regulatory mechanisms of miR-98 on cells, we predicted putative targets of miR-98 by bioinformatic analysis and found that growth and differentiation factor 6 (GDF6) and phosphatidylinositol-4-phosphate adaptor protein-2 (FAPP2) were highly conserved among different species. In consideration of the abundant expression of GDF6 and FAPP2 in placenta, we chose them for further investigation (Fagerberg et al. 2014). GDF6 and FAPP2 3'-UTR-mediated luciferase activity is specifically responsive to transfection of miR-98 mimic and inhibitor. And mutation of seed sequence in the 3'-UTR of GDF6 and FAPP2 significantly reduced the binding ability to miR-98. Moreover, miR-98 inversely regulated endogenous GDF6 and FAPP2 expression. The 
A
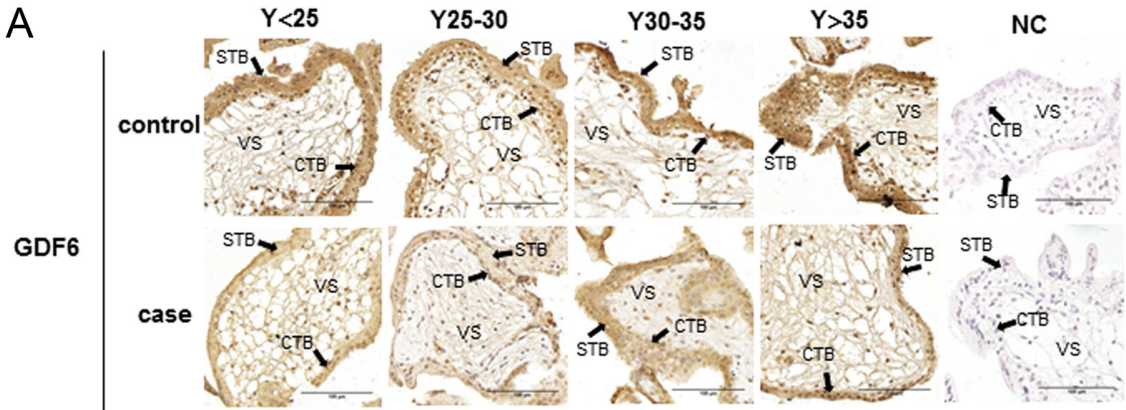

FAPP2
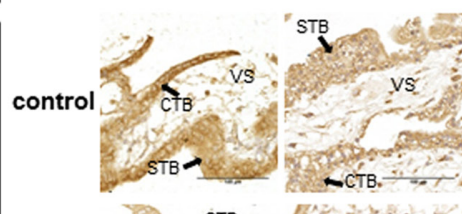

case
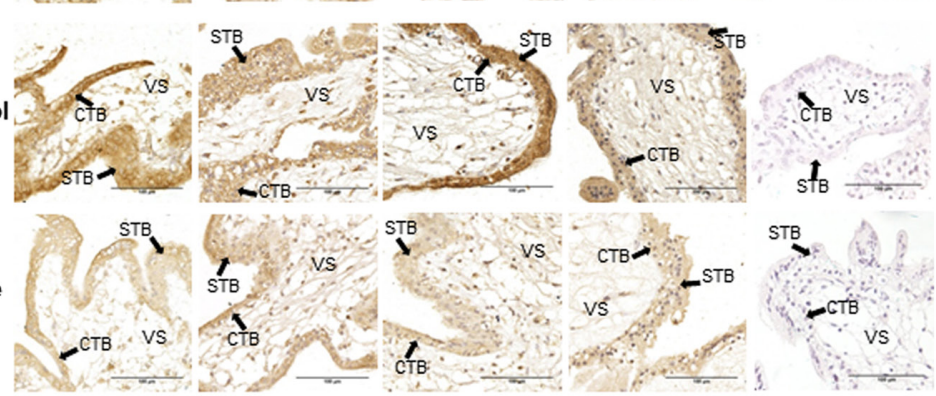

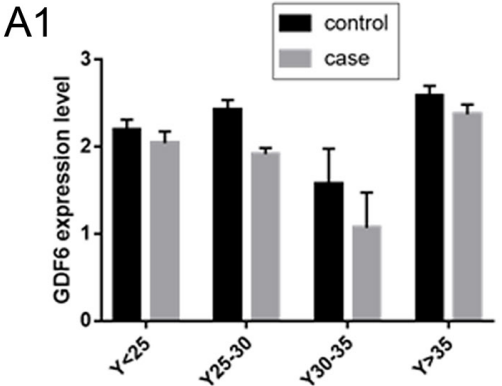

A2

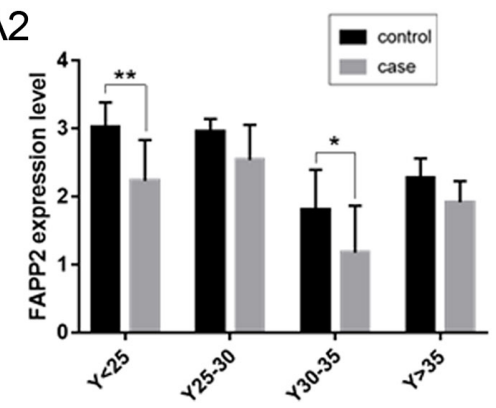

Figure 9 The expression of GDF6 and FAPP2 in placental tissues. (A) The expression of GDF6 and FAPP2 in MA placental tissues and normal placental tissues was analyzed by immunohistochemistry. The sections were incubated with fetal bovine serum as negative control. The stain was developed with DAB and characterized by yellow-brown positive signal. The scale bar is $100 \mu \mathrm{m}$. CTB: cytotrophoblast; STB: syncytiotrophoblast; VS: villous stroma. The gene expression is reflected by the intensity of positive signals quantitatively analyzed using the ImageJ soft. (A1) The comparison of GDF6 expression level between MA placental tissues (case group) and normal placental tissues (control group) in different age groups. (A2) Statistics of FAPP2 expression in different age groups. ${ }^{*} P<0.05,{ }^{* *} P<0.01$.

down-regulation of miR-98-mediated the enhancement of cell growth and invasion and was partially weakened by knockdown of GDF6 or FAPP2. All these facts show that GDF6 and FAPP2 are the functional target gene of miR-98.

GDF6 governing an embryonic cell gene signature has been shown to play an important role in embryonic skeletogenesis, organogenesis and nervous system development (Settle et al. 2003, Nohe et al. 2004, Seemann et al. 2009, Bragdon et al. 2011, Hegarty et al. 2013, Yadin et al. 2016). Gdf6a(-/-) mutants displayed maximal ocular apoptosis and induced microphthalmia based on the regulation of retinal progenitor cell number and proliferation in the ciliary marginal zone at 28 hours post fertilization (hpf) (French et al. 2013, Pant et al. 2013). In addition, GDF6 have been implicated in limb joint formation and chondrogenesis in mammals (Chang et al. 1994, Thomas et al. 1996). It prevents differentiation of coronal suture mesenchyme in early cranial development and also has an essential role in retinal development (Asai-Coakwell et al. 2007, Clendenning \& Mortlock 2012). Although the relationship between GDF6 and abortion has not been reported so far, all the previously mentioned findings suggest that GDF6 plays an important role in embryo development. High rates of recurrent pregnancy loss/ MA were observed in a majority of embryos presenting with recurrent abnormal morphology (Philipp et al. 2003, Feichtinger et al. 2018). Therefore, we speculated that the up-regulation of miR-98 in MA inhibited the expression of GDF6, which caused abnormal embryonic development and even death, and then result in MA.

FAPP2 is a phosphoinositide receptor protein and a glycolipid transporter, which plays a key role in the production of glycosphingolipids (GSLs). FAPP2 overexpression could promote colon cancer cell growth through activation of $\mathrm{Wnt} / \beta$-catenin signaling (Chen et al. 2019). Wnt/ $\beta$-catenin pathway has been confirmed to regulatecellfatedetermination, differentiation, proliferation and apoptosis during embryonic development, blastocyst activation for implantation and uterine decidualization. While aberrant Wnt signaling can damage embryo implantation and decidualization processes (Katoh \& Katoh 2006, Zhang \& Yan 2016). The downregulation of FAPP2 gene could increase tumor cell sensitivity to Fasinduced apoptosis (Tritz et al. 2009). These results imply that FAPP2 can positively regulate tumor cell invasion. Trophoblast invasion during embryo implantation in some aspects resembles tumor cell invasion. Our research demonstrated that FAPP2 expression had an obvious downward trend in villus tissues of MA patients and that miR-98 oppositely controlled FAPP2 expression. On the basis of the previously mentioned results, we speculated that high-expression of miR-98 in villus tissues of MA patients restrained trophoblast proliferation and invasion by inhibiting FAPP2 expression, which might lead to abnormal expression of $\mathrm{Wnt} / \beta$-catenin signaling and Fas/FasL signaling. These events may result in a shallow of 
embryo implantation and abnormal decidualization and finally cause MA.

miRNAs have become an ideal class of biomarker candidates for clinical diagnosis and treatment based on miRNAs stability and tissue specificity. Here, we found that $m i R-98$ level was significantly increased in $Y>35$ MA patients. Therefore, we considered to administer miR-98 inhibitor or compensate miR-98 target genes, GDF6 and FAPP2, to treat miR-98-positive MA patients.

Taken together, our results demonstrate that up-regulation of miR-98 is associated with the occurrence of MA. miR-98 prevents proliferation, viability and migration of trophoblast cells by regulating GDF6 and FAPP2. This study may provide new insights into the pathogenesis of MA.

\section{Supplementary materials}

This is linked to the online version of the paper at https://doi. org/10.1530/REP-19-0282.

\section{Declaration of interest}

The authors declared that there is no conflict of interest that could be perceived as prejudicing the impartiality of the research reported.

\section{Funding}

This work was funded by the National Natural Science Foundation of China (No. 81771590), The National Key Research, Development Program of China (2016YFC1000307) and CAMS Innovation Fund for Medical Sciences (CIFMS, 2018-I2M-1-004).

\section{Author contribution statement}

H F X and X M conceived and designed the experiments. Q Z, $Y C D$ and $L Z$ performed the experiments. Q $Z$ analyzed the data. Q Z and H F X wrote the paper. HFX and XM performed the final editing of the text.

\section{Acknowledgments}

The authors are grateful to Prof Yanling Wang from the Institute of Zoology of Chinese Academy of Sciences for gifting the HTR8/SVneo cells.

\section{References}

Asai-Coakwell M, French CR, Berry KM, Ye M, Koss R, Somerville $M$, Mueller R, Van Heyningen V, Waskiewicz AJ \& Lehmann OJ 2007 GDF6, a novel locus for a spectrum of ocular developmental anomalies. American Journal of Human Genetics 80 306-315. (https://doi. org/10.1086/511280)

Bragdon B, Moseychuk O, Saldanha S, King D, Julian J \& Nohe A 2011 Bone morphogenetic proteins: a critical review. Cellular Signalling $\mathbf{2 3}$ 609-620. (https://doi.org/10.1016/j.cellsig.2010.10.003)
Budwit-Novotny DA, McCarty KS, Cox EB, Soper JT, Mutch DG, Creasman WT, Flowers JL \& McCarty Jr KS 1986 Immunohistochemical analyses of estrogen receptor in endometrial adenocarcinoma using a monoclonal antibody. Cancer Research 46 5419-5425.

Bulletti C, Flamigni C \& Giacomucci E 1996 Reproductive failure due to spontaneous abortion and recurrent miscarriage. Human Reproduction Update 2 118-136. (https://doi.org/10.1093/humupd/2.2.118)

Chang SC, Hoang B, Thomas JT, Vukicevic S, Luyten FP, Ryba NJ, Kozak CA, Reddi AH \& Moos Jr M 1994 Cartilage-derived morphogenetic proteins. New members of the transforming growth factor-beta superfamily predominantly expressed in long bones during human embryonic development. Journal of Biological Chemistry $26928227-28234$.

Chen J, Li L, Zhou Z, Yu S, Li Y \& Gao Y 2019 FAPP2 promotes tumor cell growth in human colon cancer through activation of Wnt signaling. Experimental Cell Research 374 12-18. (https://doi.org/10.1016/j. yexcr.2018.11.002)

Clendenning DE \& Mortlock DP 2012 The BMP ligand Gdf6 prevents differentiation of coronal suture mesenchyme in early cranial development. PLOS ONE 7 e36789. (https://doi.org/10.1371/journal. pone.0036789)

Clifford K, Rai R, Watson H \& Regan L 1994 An informative protocol for the investigation of recurrent miscarriage: preliminary experience of 500 consecutive cases. Human Reproduction 9 1328-1332. (https://doi. org/10.1093/oxfordjournals.humrep.a138703)

Cohen M, Wuillemin C, Irion O \& Bischof P 2010 Role of decidua in trophoblastic invasion. Neuro Endocrinology Letters 31 193-197.

Fagerberg L, Hallström BM, Oksvold P, Kampf C, Djureinovic D, Odeberg J, Habuka M, Tahmasebpoor S, Danielsson A, Edlund K et al. 2014 Analysis of the human tissue-specific expression by genome-wide integration of transcriptomics and antibody-based proteomics. Molecular and Cellular Proteomics 13 397-406. (https://doi.org/10.1074/mcp. M113.035600)

Feichtinger M, Reiner A, Hartmann B \& Philipp T 2018 Embryoscopy and karyotype findings of repeated miscarriages in recurrent pregnancy loss and spontaneous pregnancy loss. Journal of Assisted Reproduction and Genetics 35 1401-1406. (https://doi.org/10.1007/s10815-018-1226-1)

French CR, Stach TR, March LD, Lehmann OJ \& Waskiewicz AJ 2013 Apoptotic and proliferative defects characterize ocular development in a microphthalmic BMP model. Investigative Ophthalmology and Visual Science 54 4636-4647. (https://doi.org/10.1167/iovs.13-11674)

Fu Y, Liu X, Chen Q, Liu T, Lu C, Yu J, Miao Y \& Wei J 2018 Downregulated miR-98-5p promotes PDAC proliferation and metastasis by reversely regulating MAP4K4. Journal of Experimental and Clinical Cancer Research 37 130. (https://doi.org/10.1186/s13046-018-0807-2)

Girard M, Jacquemin E, Munnich A, Lyonnet S \& Henrion-Caude A 2008 miR-122, a paradigm for the role of microRNAs in the liver. Journal of Hepatology 48 648-656. (https://doi.org/10.1016/j.jhep.2008.01.019)

Hatasaka HH 1994 Recurrent miscarriage: epidemiologic factors, definitions, and incidence. Clinical Obstetrics and Gynecology 37 625-634. (https://doi.org/10.1097/00003081-199409000-00016)

Hegarty SV, O'Keeffe GW \& Sullivan AM 2013 BMP-Smad 1/5/8 signalling in the development of the nervous system. Progress in Neurobiology 109 28-41. (https://doi.org/10.1016/j.pneurobio.2013.07.002)

Hromadnikova I, Kotlabova K, Doucha J, Dlouha K \& Krofta L 2012 Absolute and relative quantification of placenta-specific microRNAs in maternal circulation with placental insufficiency-related complications. Journal of Molecular Diagnostics 14 160-167. (https://doi.org/10.1016/j. jmoldx.2011.11.003)

Hwang SU, Jeon Y, Yoon JD, Cai L, Kim E, Yoo H, Kim KJ, Park KM, Jin M, Kim H et al. 2015 Effect of ganglioside GT1b on the in vitro maturation of porcine oocytes and embryonic development. Journal of Reproduction and Development 61 549-557. (https://doi.org/10.1262/ jrd.2015-049)

Kafil N \& Arain F 2017 Comparison of efficacy of vaginal vs oral prostaglandin E1 analogue (misoprostol) in management of first trimester missed abortion. Medical Forum Monthly 28 45-49. (https://doi. org/10.7860/jcdr/2016/18178.7891)

Katoh Y \& Katoh M 2006 Comparative integromics on BMP/GDF family. International Journal of Molecular Medicine 17 951-955. (https://doi. org/10.3892/ijmm.17.5.951)

Lewis BP, Burge CB \& Bartel DP 2005 Conserved seed pairing, often flanked by adenosines, indicates that thousands of human genes are microRNA targets. Cell 120 15-20. (https://doi.org/10.1016/j.cell.2004.12.035) 
Li H, Guo L, Wu Q, Lu J, Ge Q \& Lu Z 2012 A comprehensive survey of maternal plasma miRNAs expression profiles using high-throughput sequencing. Clinica Chimica Acta 413 568-576. (https://doi. org/10.1016/j.cca.2011.11.026)

Long M, Zhan M, Xu S, Yang R, Chen W, Zhang S, Shi Y, He Q, Mohan M, Liu Q et al. 2017 miR-92b-3p acts as a tumor suppressor by targeting Gabra3 in pancreatic cancer. Molecular Cancer 16 167. (https://doi. org/10.1186/s12943-017-0723-7)

Ni R, Huang Y \& Wang J 2015 miR-98 targets ITGB3 to inhibit proliferation, migration, and invasion of non-small-cell lung cancer. OncoTargets and Therapy 8 2689-2697. (https://doi.org/10.2147/OTT.S90998)

Nohe A, Keating E, Knaus P \& Petersen NO 2004 Signal transduction of bone morphogenetic protein receptors. Cellular Signalling 16 291-299. (https://doi.org/10.1016/j.cellsig.2003.08.011)

Pant SD, March LD, Famulski JK, French CR, Lehmann OJ \& Waskiewicz AJ 2013 Molecular mechanisms regulating ocular apoptosis in zebrafish gdf6a mutants. Investigative Ophthalmology and Visual Science 54 5871-5879. (https://doi.org/10.1167/iovs.12-11315)

Philipp T, Philipp K, Reiner A, Beer F \& Kalousek DK 2003 Embryoscopic and cytogenetic analysis of 233 missed abortions: factors involved in the pathogenesis of developmental defects of early failed pregnancies. Human Reproduction 18 1724-1732. (https://doi.org/10.1093/humrep/ deg309)

Reinhart BJ, Slack FJ, Basson M, Pasquinelli AE, Bettinger JC, Rougvie AE, Horvitz HR \& Ruvkun G 2000 The 21-nucleotide let-7 RNA regulates developmental timing in Caenorhabditis elegans. Nature 403 901-906. (https://doi.org/10.1038/35002607)

Salic A \& Mitchison TJ 2008 A chemical method for fast and sensitive detection of DNA synthesis in vivo. PNAS 105 2415-2420. (https://doi. org/10.1073/pnas.0712168105)

Seemann P, Brehm A, König J, Reissner C, Stricker S, Kuss P, Haupt J, Renninger S, Nickel J, Sebald W et al. 2009 Mutations in GDF5 revea a key residue mediating BMP inhibition by NOGGIN. PLoS Genetics 5 e1000747. (https://doi.org/10.1371/journal.pgen.1000747)

Settle Jr SH, Rountree RB, Sinha A, Thacker A, Higgins K \& Kingsley DM 2003 Multiple joint and skeletal patterning defects caused by single and double mutations in the mouse Gdf6 and Gdf5 genes. Developmental Biology 254 116-130. (https://doi.org/10.1016/s0012-1606(02)00022-2)

Thomas JT, Lin K, Nandedkar M, Camargo M, Cervenka J \& Luyten FP 1996 A human chondrodysplasia due to a mutation in a TGF-beta superfamily member. Nature Genetics 12 315-317. (https://doi. org/10.1038/ng0396-315)

Tritz R, Hickey MJ, Lin AH, Hadwiger P, Sah DW, Neuwelt EA, Mueller BM \& Kruse CA 2009 FAPP2 gene downregulation increases tumor cell sensitivity to Fas-induced apoptosis. Biochemical and Biophysical Research Communications 383 167-171. (https://doi.org/10.1016/j. bbrc.2009.03.126)

Wang Y, Lv Y, Wang L, Gong C, Sun J, Chen X, Chen Y, Yang L, Zhang Y, Yang $\mathbf{X}$ et al. 2015 MicroRNAome in decidua: a new approach to assess the maintenance of pregnancy. Fertility and Sterility 103 980.e6-989.e6. (https://doi.org/10.1016/j.fertnstert.2015.01.003)

Wei A, Shen B, Williams LA, Bhargav D, Gulati T, Fang Z, Pathmanandavel S \& Diwan AD 2016 Expression of growth differentiation factor 6 in the human developing fetal spine retreats from vertebral ossifying regions and is restricted to cartilaginous tissues. Journal of Orthopaedic Research 34 279-289. (https://doi.org/10.1002/jor.22983)

Xia HF, Jin XH, Cao ZF, Shi T \& Ma X 2014 MiR-98 is involved in rat embryo implantation by targeting Bcl-xl. FEBS Letters 588 574-583. (https://doi.org/10.1016/j.febslet.2013.12.026)

Xiao Q, Zeng FL, Tang GY, Lei CY, Zou XX, Liu XL, Peng BL, Qin S \& Li HX 2019 Expression of galectin-3 and apoptosis in placental villi from patients with missed abortion during early pregnancy. Experimental and Therapeutic Medicine 17 2623-2631. (https://doi.org/10.3892/ etm.2019.7227)

Yadin D, Knaus P \& Mueller TD 2016 Structural insights into BMP receptors: specificity, activation and inhibition. Cytokine and Growth Factor Reviews 27 13-34. (https://doi.org/10.1016/j.cytogfr.2015.11.005)

Zhang Q \& Yan J 2016 Update of Wnt signaling in implantation and decidualization. Reproductive Medicine and Biology 15 95-105. (https://doi.org/10.1007/s12522-015-0226-4)

Zhang X, Li J, Gu Y, Zhao Y, Wang Z \& Jia G 2011 A pilot study on environmental and behavioral factors related to missed abortion. Environmental Health and Preventive Medicine 16 273-278. (https://doi. org/10.1007/s12199-010-0196-4)

Zhu Y, Lu H, Huo Z, Ma Z, Dang J, Dang W, Pan L, Chen J \& Zhong H 2016 MicroRNA-16 inhibits feto-maternal angiogenesis and causes recurrent spontaneous abortion by targeting vascular endothelial growth factor. Scientific Reports 6 35536. (https://doi.org/10.1038/srep35536)

Received 29 June 2019

First decision 21 August 2019

Revised manuscript received 15 January 2020

Accepted 10 February 2020 